\title{
The Role of Fnac in Diagnosis of Papillary Carcenoma of Thyroid
}

\author{
Dr.Ali Nyyef , Dr.Alaa Jamel Hassan, Dr.Rasha Kussai Aljaoha \\ Assi (C.A.B.S) Medical College Thiqar Univrsitey \\ (Fibms Path) Medical College Thiqar University \\ (C.A.B.S) Medical College Thi-Qar University
}

\begin{abstract}
Fine-needle aspiration of the thyroid gland is safe, inexpensive, minimally invasive, and highly accurate in the diagnosis of nodular thyroid disease. Limitations of FNAC are related to the skill of the aspirator, the experience of the cytologist, and the difficulty in distinguishing some benign cellular adenomas from their malignant counterpart ${ }^{[1]}$.Papillary thyroid cancer or papillary thyroid carcinoma is the most common type of thyroid cancer, representing $75 \%$ to $85 \%$ of all thyroid cancer cases. ${ }^{[1][2]}$ It occurs more frequently in women and commonly between 30-40 years. It is also the predominant type thyroid cancer in children, especially in patients who had previous radiation to the head and neck. ${ }^{[3]}$ This study was conducted in Al Hussein teaching hospital through 5 years from 2008 to 2013 on 300 patients complained from thyroid nodules , FNAC performed to these patients who suspected to have thyroid malignancy from history and clinical examination, 52 patients( $17.3 \%)$ was diagnosed as a papillary carcinoma,40patients were females $(76.2 \%)$ and 12 were males (23.8\%), ages ranged between $28-46$ years, follow up of these patients with histopathplogical examination to confirm the diagnosis. After histopathological examination we get 41 cases(78.8\%) were confirmed as apapillary carcinomas (32 cases were females and 9 males cases. ), 6 patients (11.5\%) diagnosed nodular hyperplasia , 2 cases (3.8\%) of follicular adenoma and 3 cases(5.7\%) of thyroiditis. 15 patients (36.5\%) of these 41 cases were from areas proved to be exposed to radiation through 1991 \& 2003 wars, 11 patients (26.8\%) had positive family history of thyroid malignancies, 9 patients (21.9\%) from radiation exposed area \& had family history of thyroid tumors and 5 cases $(12.2 \%)$ had negative history \& one case with long standing $M N G$.
\end{abstract}

Key Words: Papillary thyroid carcinoma, FNAC

\section{Introduction:}

FNAC of the thyroid gland is safe, inexpensive, minimally invasive, and highly accurate in the diagnosis of nodular thyroid disease. Four cytological diagnostic categories are used, rates for these categories based on data pooled from seven series, were as follows: benign 69\%; suspicious $10 \%$; malignant 4\%; and no diagnostic 17\%. Analysis of recent data suggests a false-negative rate of 1 $11 \%$, a false-positive rate of $1-8 \%$, a sensitivity of $65 \%$ to $98 \%$, and a specificity of $72 \%$ to $100 \%{ }^{[1]}$ Limitations of FNAC are related to the skills of the aspirator, the experience of the cytologist, and the difficulty in distinguishing some benign cellular adenomas from their malignant counterparts. ${ }^{[3]}$ The introduction of fine-needle aspiration has had a substantial effect on the management of patients with thyroid nodules. The percentage of patients undergoing thyroidectomy has decreased by $25 \%$, and the yield of carcinoma in patients who undergo surgery has increased from $15 \%$ to at least $30 \%$. Fine-needle aspiration has decreased the cost of care by $25 \%{ }^{[2]}$ Should fine needle aspiration biopsy be done on all thyroid nodules? There are certain situations in which your physician may elect not to perform a FNA of a nodule. For example, in a patient with an over- active thyroid (hyperthyroidism), the chance for a nodule to be cancerous is significantly less; particularly if other studies (such as nuclear thyroid imaging) show that the nodule is producing thyroid hormone (a "hot" nodule). ${ }^{[4]}$

Fine needle aspiration cytology of the thyroid nodule recommend in the following situations:

- To make a diagnosis of a thyroid nodule.

- To help select therapy for a thyroid nodule.

- To drain a cyst that may be causing pain.

- To inject a medication to shrink a recurrent cyst. ${ }^{[4]}$

How fine needle aspiration cytology performed?

In most cases, if the nodule can be felt, FNA can be performed in the doctor's office. In some cases an ultrasound may be needed to help guide the aspiration. For example, if the nodule cannot be felt or if the nodule has areas within it that specifically should be aspirated. Little preparation by the patient is required. There is no need to fast or to withhold medications on the day of the aspiration. Occasionally, though, a patient may be asked not to take blood thinning medication on the day of the 
biopsy. After an examination to pinpoint the nodule, the patient is asked to lie down and the neck is exposed. Depending on the location of the nodule and the types of clothes the patient is wearing, he or she may be asked to change into a gown. The doctor drapes the area around the neck and cleans the neck off. This is usually done with iodine, which is a brown liquid that sterilizes the skin. Some doctors may choose to inject a local anesthetic. Often, the injection of the anesthetic results in an initial discomfort, like a bee sting. The majority of doctors who regularly perform fine needle aspiration of the thyroid do not use a local anesthetic for this reason. Since the needle used for fine needle aspiration is so fine, anesthesia often results in simply another uncomfortable poke for the patient. If a patient is particularly concerned and nervous, a topical anesthetic preparation may be applied, which takes 10 to 20 minutes to work, thus prolonging the procedure. ${ }^{[5]} \mathrm{A}$ patient undergoing fine needle aspiration should discuss any preferences for local anesthetic before the procedure begins. Most patients undergoing fine needle aspiration foregot the use of any anesthetic and do very well. ${ }^{[4]}$ Once the patient is ready, a small, fine-gauge needle is inserted into the nodule. The needle is smaller in diameter than the needle used in most blood draws (usually a 25 gauge 1.5 inch needle). The patient holds his breath while the needle is rocked gently to obtain as much tissue as possible. (The reason for holding the breath is to minimize movement of the structures in the neck.) The needle is then withdrawn and pressure is applied over the thyroid area to minimize bleeding. This procedure is usually repeated four to six times to ensure that an adequate amount of tissue has been collected. After the procedure, pressure is applied over the neck area for 5 to 10 minutes to assure that the bleeding has stopped. The pressure also helps to reduce any swelling that may occur. The entire procedure usually takes less than 20 minutes. ${ }^{[5]}$

The Following Features Increase the Suspicion of a Malignant Nodule:

- Age less than 20

- $\quad$ Age greater than 70

- Male gender

- New onset of swallowing difficulties

- New onset of hoarseness

- History of external neck irradiation during childhood

- Firm, irregular, and fixed nodule

- Presence of cervical lymphadenopathy (swollen, hard lymph nodes in the neck)

- Previous history of thyroid cancer

- Nodule that is "cold" on scan (shown in picture above, meaning the nodule does not make hormone)

- Solid or complex on an ultrasound. ${ }^{[6]}$

Papillary thyroid cancer or papillary thyroid carcinoma is the most common type of thyroid cancer representing $75 \%$ to $85 \%$ of all thyroid cancer cases. ${ }^{[1][2]}$ It occurs more frequently in women and presents in the 30-40 years age group. It is also the predominant type of thyroid cancer in children, and in patients who had previous radiation to the head and neck. ${ }^{[3]}$. Thyroglobulin can be used as a tumor marker for well-differentiated papillary thyroid cancer. ${ }^{[4][5]}$ HBME-1 staining may be useful for differentiating papillary carcinomas from follicular carcinomas; in papillary lesions it tends to be positive. ${ }^{[6]}$

Pathology

- Characteristic Orphan Annie eye nuclear inclusions (nuclei with uniform staining, which appear empty $)^{[7]}$ and psammoma bodies on light microscopy. The former is useful in identifying the follicular variant of papillary thyroid carcinomas. ${ }^{[8]}$

- Lymphatic spread is more common than hematogenous spread, multifocality is common ,the socalled Lateral Aberrant Thyroid is actually a lymph node metastasis from papillary thyroid carcinoma. ${ }^{[9]}$

- Papillary micro carcinoma is a subset of papillary thyroid cancer defined as measuring less than or equal to $1 \mathrm{~cm} \cdot{ }^{[10]}$ The highest incidence of papillary thyroid micro carcinoma in an autopsy series was reported by Harach et al. in 1985, who found 36 of 101 consecutive autopsies to have an incidental micro carcinoma. ${ }^{[1]}$ Michael Pakdaman et al. report the highest incidence in a retrospective surgical series at $49.9 \%$ of 860 cases. $^{[12]}$ Management strategies for incidental papillary micro carcinoma on ultrasound (and confirmed on FNAB) range from total thyroidectomy with radioactive iodine ablation to observation alone. Harach et al. suggest using the term "occult papillary tumor" to avoid giving patients distress over having cancer. It was Woolner et al. who first arbitrarily coined the term "occult papillary carcinoma" in 1960, to describe papillary carcinomas $\leq 1.5 \mathrm{~cm}$ in diameter. ${ }^{[13]}$. Although papillary carcinoma has a propensity to invade lymphatic, it is less likely to invade blood vessels. ${ }^{[14]}$ These kinds of tumors 
are most commonly unencapsulated, and they have a high tendency to metastasize locally to lymph nodes, which may produce cystic structures near the thyroid that are difficult to diagnose because of the paucity of malignant tissue. ${ }^{[15]}$ Furthermore, papillary tumors may metastasize to the lungs and produce a few nodules or the lung fields may exhibit a snow flake appearance throughout.Other characteristics of the papillary carcinoma is that E.M. shows increased mitochondria, increased RER, as well as increased apical microvilli. Moreover, papillary carcinomas have an indolent growth, and $40 \%$ of cases spread out of the capsule. ${ }^{[16]}$

\section{Diagnosis}

Papillary thyroid carcinoma is usually discovered on routine examination as an asymptomatic thyroid nodule that appears as a neck mass. In some instances, the mass may have produced local symptoms. This mass is normally referred to FNA for investigation. FNA accuracy is very high and it is a process widely used in these cases. Other investigation methods include ultrasound imaging and nuclear scan. The ultrasound is a useful test to distinguish solid from cystic lesions and to identify calcifications. ${ }^{[17]}$ The thyroid ultrasound is effective to discover micro carcinomas, which refer to very small carcinomas $(<1 \mathrm{~cm})$.Papillary thyroid carcinomas are also discovered when a hard nodule is found in multinodular goiter, when enlarged cervical lymph nodes are detected, or when there are unidentified metastatic lesions elsewhere in the body. ${ }^{[15]}$ Expanding lesions found in the thyroid gland, especially if they are painful, should be examined as they may indicate the presence of papillary thyroid carcinoma. Other clinical signs that could indicate papillary thyroid are: fixation to the trachea, stony hardness, damage to recurrent laryngeal or cervical sympathetic nerves. Seventy five percent of the population will have these thyroid nodules, and the majority will always be benign. ${ }^{[18]}$. Chia et al. report that TSHR mRNA measured with FNA enhances the preoperative detection of cancer in patients with thyroid nodules, reducing unnecessary surgeries, and immediate postoperative levels can predict residual/metastatic disease ${ }^{[15]}$ Suspected lung metastases and/or miliary aspects at $\mathrm{x}$-ray or $\mathrm{CT}$ can be diagnosed by cytology on a broncho alveolar lavage specimen. ${ }^{[19]}$

aim of study:

To know the role of FNAC in diagnosis of papillary carcinoma in thyroid nodules and the prevalence of this type of carcinoma compared with other types of thyroid carcinoma in Thiqar city.

\section{Patient \& Methods:}

This study was conducted in AL-Hussein teaching hospital and private labs. through 5 years from January 2008 to January 2013 on 300 patients complaining from thyroid nodules who suspected to have thyroid malignancy from history and clinical examination. FNAC were performed for these patients, the results of 52 patients(17.3\%) were positive for papillary carcinoma, 12 males and 40 females, age ranged from 28-52 years. Positive FNAC results for papillary carcinoma depend on cytological criteria as hyper cellularity, papillary configuration and nuclear feature of large hyper chromatic nuclei, and opened chromatin ${ }^{[16]}$, follow up of those 52 patients by total thyroidectomy or near total thyroidectomy after explained to the patients including the intra-operative and postoperative complications that may occur and took concept of operation, the specimen sent for histopathological examination which are stained by routine H\&E stain review by 2 histopathologist and compared the 2 histopathological results .

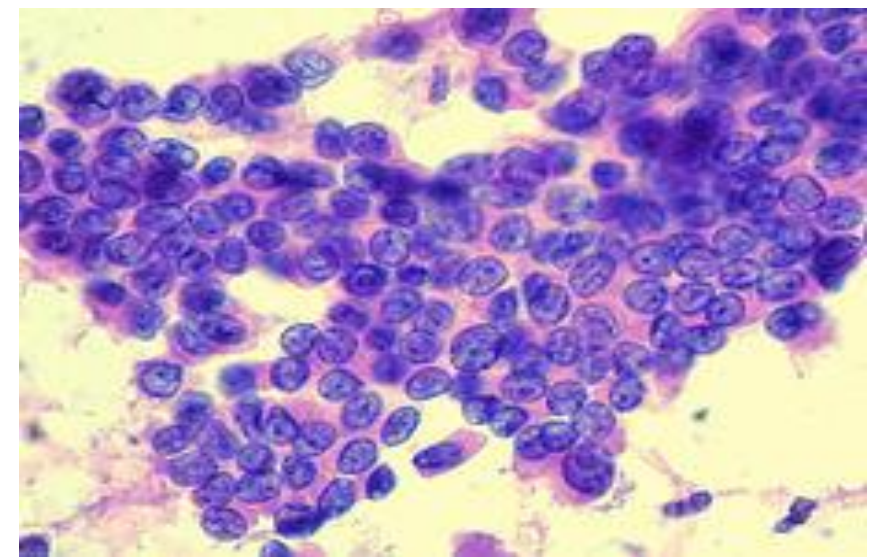

Finding of cytological examination of papillary thyroid carcinoma 
These are some pathological picture for papillary thyroid carcinoma that appear in our study.

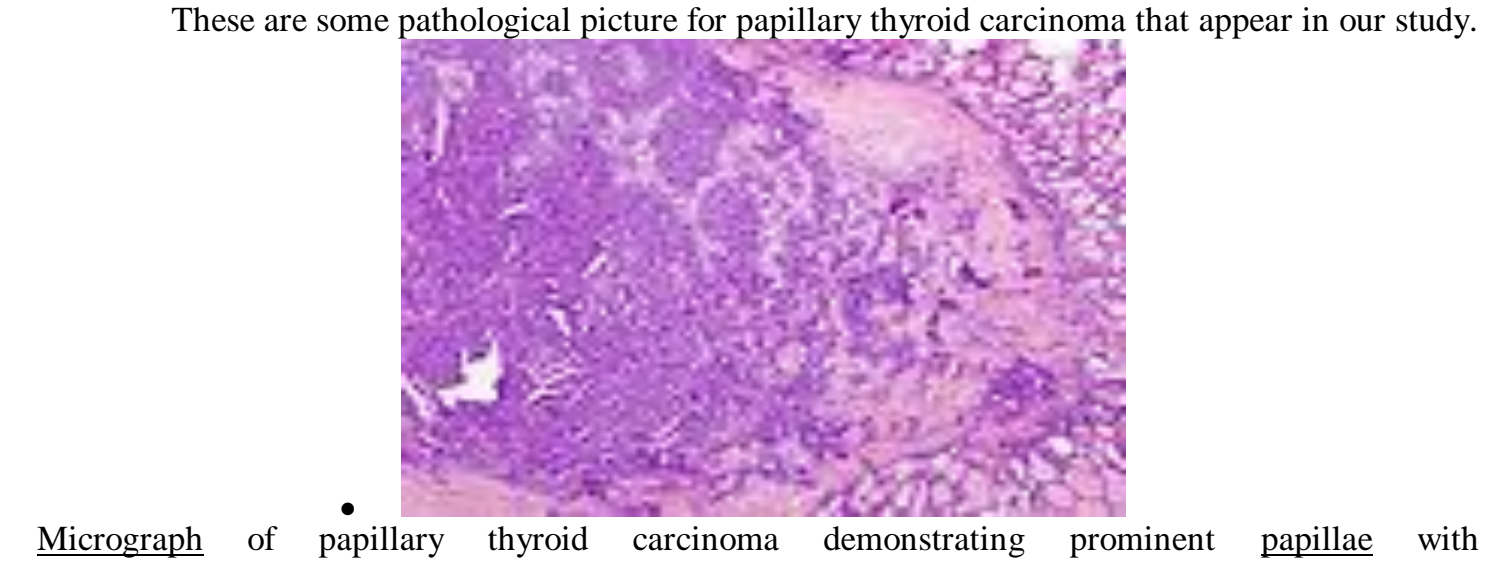
fibrovascular cores. $\underline{H \& E \text { stain. }}$.

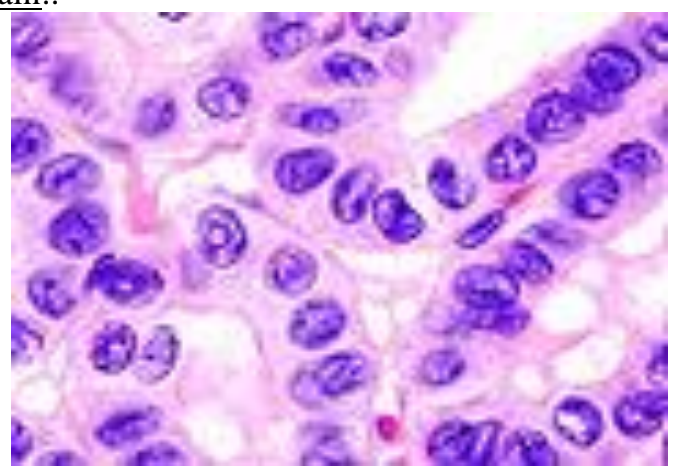

Micrograph (high power view) showing nuclear changes in papillary thyroid carcinoma (PTC), which include groove formation, optical clearing, eosinophilic inclusions and overlapping of nuclei. H\&E stain.

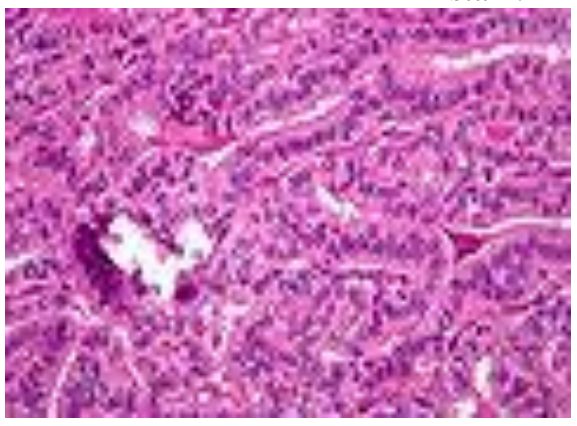

$\underline{\text { Micrograph of papillary thyroid carcinoma, tall cell variant - high magnification. } \underline{H \& E} \text { stain. }}$

\section{Results:}

From FNAC studies on 300 patients who suspected to have thyroid malignancy, 52 cases (17.3\%) had papillary carcinoma, 40 were females (76.2\%) and 12 male patients (23.8\%). Age ranging between 28-52 years. 17cases (5.6\%) follicular adenoma, 3 cases (1\%) medullary carcinoma , 2 cases $(0.6 \%)$ anaplastic carcinoma , 226 cases $(75 \%)$ were thyroid hyperplasia \& other benign lesions ,those 52 cases who have positive results for papillary carcinoma on FNAC near total or total thyroidectomy performed \& send for histopathological examination , 41(78.8\%) from those 52 cases the diagnosis confirmed, 6 cases $(11.5 \%)$ nodular hyperplasia, $2(3.8 \%)$ follicular adenoma and 3 cases $(5.7 \%)$ were one types of thyroiditis, 15 patients $(36.5 \%)$ of these 41 cases were from areas proved to be exposed to radiation through 1991 \& 2003 wars , 11 patients (26.8\%) had positive family history of thyroid malignancies, 9 patients $(21.9 \%)$ from radiation exposed area \& had family history of thyroid tumors and 5 cases $(12.2 \%)$ have negative history \& one case with long standing MNG. $32(78.1 \%)$ cases were females and $9(21.9 \%)$ case were males. 


\section{Discussion :}

FNAC of the thyroid gland is safe, inexpensive, minimally invasive, and highly accurate in the diagnosis of nodular thyroid disease, so FNAC is recommended for any patient complaining from thyroid nodule as early as possible for early detection of thyroid cancer for selection of proper surgical procedure to improve the survival rate with better prognostic outcome. Our study found that the papillary carcinoma was the commonest thyroid malignancy and contributed to $17 \%$ of cases $(52$ patients from 300 patients who had clinical suspicion of thyroid malignancy) have positive FNAC results ,But after histopathological examination 41 patients (78\%) proved the diagnosis which means that FNAC have high accurate rate with about $20 \%$ false positive result so our study differ from many books which said the false positive result of FNAC is $1-9 \%$, also we found that the main predisposing factors for papillary thyroid carcinoma were history of radiation \& not a family history reverse to some thesis that said family history was the main predisposing factor, about age of affected patients by papillary carcinoma may be up to 52 years, few cases(less than 2.5\%) of long standing M.N.G. might developed papillary carcinoma, this type of carcinoma affected any persons even with negative history.

\section{Conclusion :}

FNAC was safe, accurate, and cost-effective. The procedure had specific role in the management of thyroid nodules and should be used as the initial diagnostic test, so FNAC recommended for most of the patients complaining from thyroid nodule unless there is a contraindication for this procedure, false positive results were about $20 \%$, this represented nearly acceptable percentage according to the characters of FNAC procedure ( safe, easy, low coast, low complication), carful history to find any predisposing factors that lead to papillary carcinoma such as history of radiation or family history though it might be negative. There was a surprising case of the papillary carcinoma occurred in patient with long standing M.N.G .

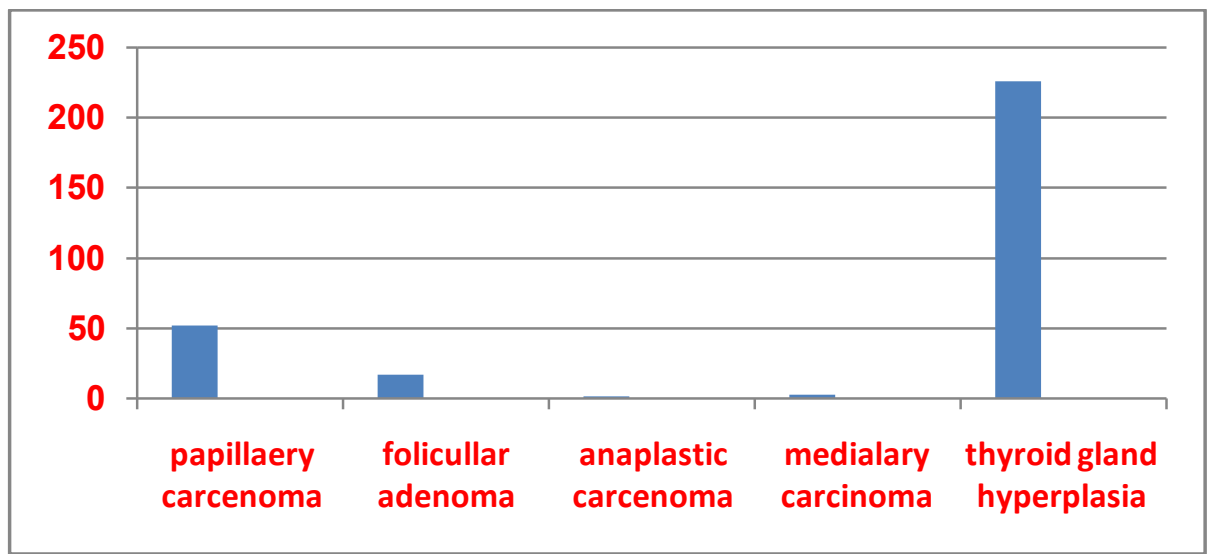

Finding of FNAC OF THYROID NODULE

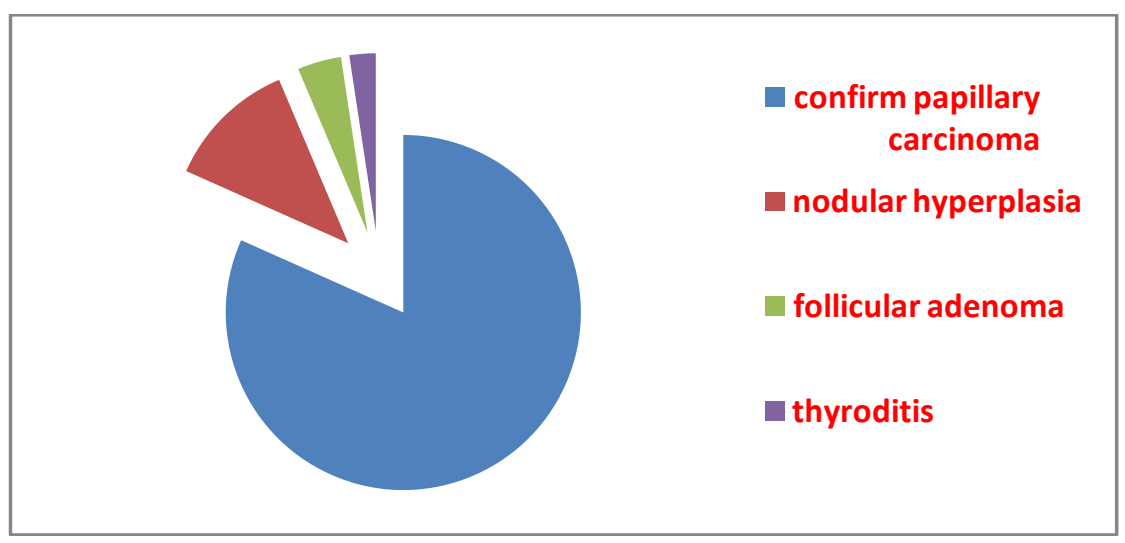

Histopathological finding to those 52 posative finding on FNAC 


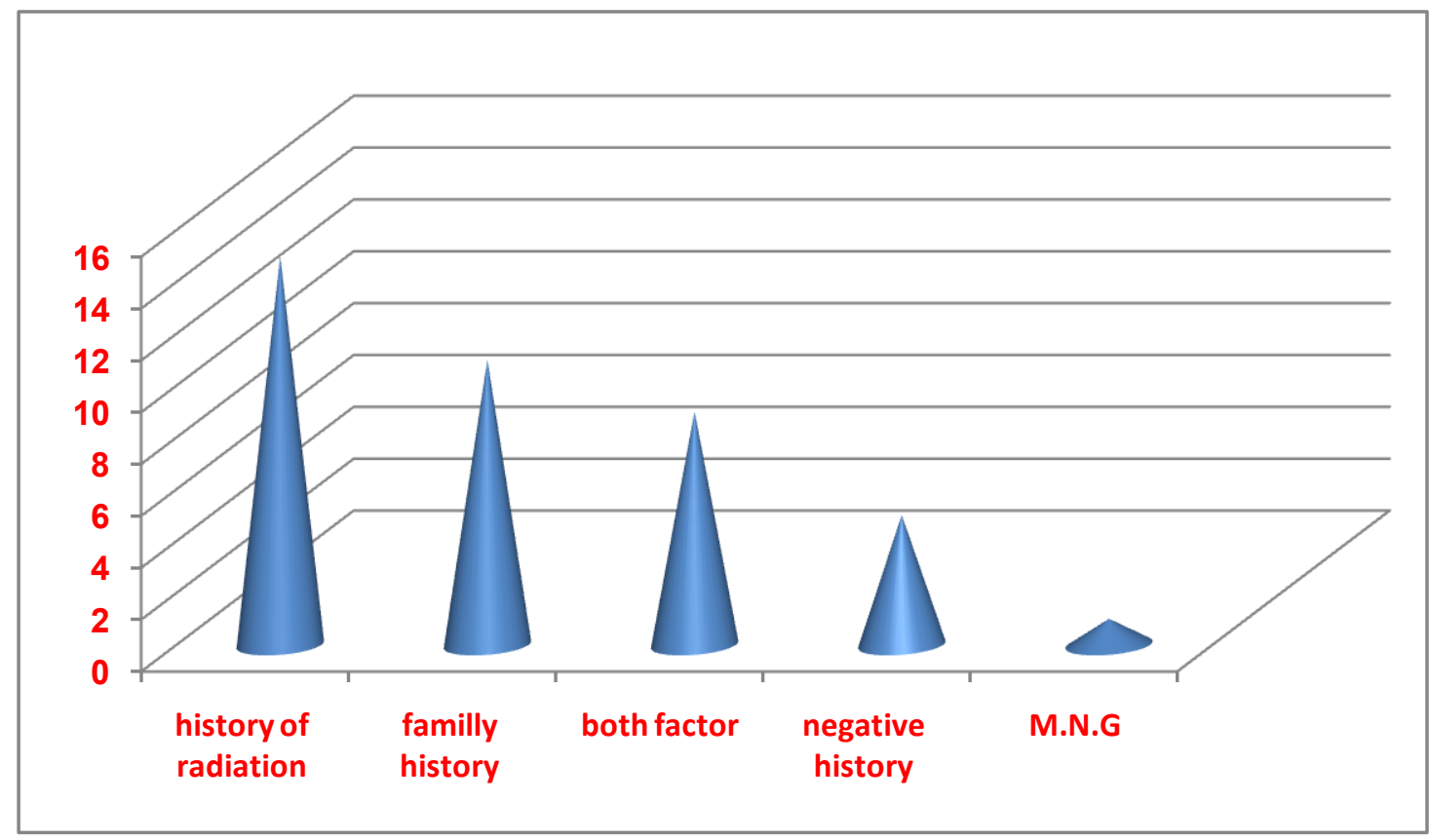

predisposing factors for papillary carcinoma

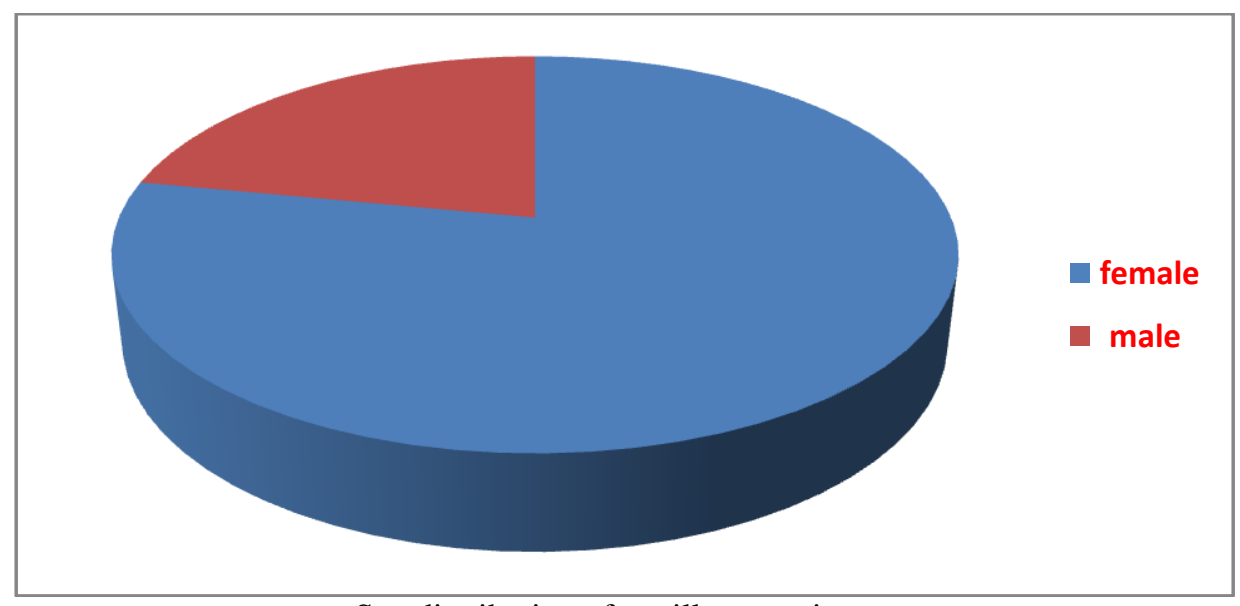

Sex distribution of papillary carcinoma

\section{References}

[1]. $\wedge \stackrel{\text { abcdef }}{ }$ Chapter 20 in: Mitchell, Richard Sheppard; Kumar, Vinay; Abbas, Abul K.; Fausto, Nelson. Robbins Basic Pathology. Philadelphia: Saunders. ISBN 1-4160-2973-7.8th edition.

[2]. $\quad \wedge$ Hu MI, Vassilopoulou-Sellin R, Lustig R, Lamont JP."Thyroid and Parathyroid Cancers" in Pazdur R, Wagman LD, Camphausen KA, Hoskins WJ (Eds) Cancer Management: A Multidisciplinary Approach.11 ed. 2008.

[3]. ^Dinets A, Hulchiy M, Sofiadis A, Ghaderi M, Höög A, Larsson C, Zedenius J. (2012). "Clinical, Genetic and Immunohistochemical Characterization of 70 Ukrainian Adult Cases with Post-Chornobyl Papillary Thyroid Carcinoma.".Eur J Endocrinol 166: 1049-60. doi: 10.1530/EJE-12-0144. PMID 22457234.

[4]. $\quad \hat{\Lambda}$ Lin JD (2007)."Thyroglobulin and human thyroid cancer".ClinChimActa 388 (1-2): 15. doi:10.1016/j.cca.2007.11.002. PMID 18060877.

[5]. $\quad$ A Tuttle RM, Leboeuf R, Martorella AJ (2007). "Papillary thyroid cancer: monitoring and therapy". Endocrinol.Metab.Clin. North $\bar{A} m .36$ (3): 753-78, vii. doi:10.1016/j.ecl.2007.04.004. PMID 17673127.

[6]. _Papotti M, Rodriguez J, De Pompa R, Bartolazzi A, Rosai J (April 2005). "Galectin-3 and HBME-1 expression in welldifferentiated thyroid tumors with follicular architecture of uncertain malignant potential".Mod.Pathol. 18 (4): 541-6. doi:10.1038/modpathol.3800321. PMID 15529186.

[7]. $\quad$ "Papillary Carcinoma of Thyroid (Hi Pow)". University of Connecticut Health Center.Retrieved 2008-09-14.

[8]. ^ Yang GC, Liebeskind D, Messina AV (2001). "Ultrasound-guided fine-needle aspiration of the thyroid assessed by Ultrafast Papanicolaou stain: data from 1135 biopsies with a two- to six-year follow-up". Thyroid 11 (6): 581-9. doi: $10.1089 / 105072501750302895$. PMID 11442006 .

[9]. ${ }^{\wedge}$ Escofet X, Khan AZ, Mazarani W, Woods WG (2007). "Lessons to be learned: a case study approach. Lateral aberrant thyroid tissue: is it always malignant?".J R Soc Health 127 (1): 45-6. doi:10.1177/1466424007073207. PMID 17319317.

[10]. ^Shaha AR (2007)."TNM classification of thyroid carcinoma".World J Surg 31 (5): 879-87. doi:10.1007/s00268-006-0864-0. PMID 17308849. 
[11]. $\triangleq$ Harach HR, Franssila KO, Wasenius VM (1985). "Occult papillary carcinoma of the thyroid. A "normal" finding in Finland.A systematic autopsy study".Cancer 56 (3): 531-8. doi:10.1002/1097-0142(19850801)56:3<531::AID-CNCR2820560321>3.0.CO;2-3. PMID 2408737.

[12]. ^Pakdaman MN, Rochon L, Gologan O, Tamilia M, Garfield N, Hier MP, Black MJ, Payne RJ (2008). "Incidence and histopathological behavior of papillary microcarcinomas: Study of 429 cases". Otolaryngol Head Neck Surg 139 (5): 718-22. doi:10.1016/j.otohns.2008.08.014. PMID 18984270.

[13]. $\wedge$ WOOLNER LB, LEMMON ML, BEAHRS OH, BLACK BM, KEATING FR (January 1960). "Occult papillary carcinoma of the thyroid gland: a study of 140 cases observed in a 30-year period". J. Clin. Endocrinol.Metab. 20: 89-105. doi:10.1210/jcem-20-1-89. PMID 13845950.

[14]. $\quad$ "Thyroid, Papillary Carcinoma".Retrieved 2010-07-15.

[15]. $\wedge$ abcd"The Thyroid and its Diseases". Retrieved 2010-07-15.

[16]. $\wedge$ "Papillary Carcinomas". Retrieved 2010-07-15. [dead link]

[17]. ^"Papillary Thyroid Carcinoma: An Overview". Archives of Pathology \& Laboratory Medicine. 2006. Retrieved 2010-07-15.

[18]. ^"Papillary Carcinoma Prognosis".Retrieved 2010-07-15.

[19]. $\wedge \underline{a b}$ Vermeer-Mens, J. C. J.; Goemaere, N. N. T.; Kuenen-Boumeester, V.; De Muinck Keizer-Schrama, S. M. P. F.; Zwaan, C. M.; Devos, A. S.; De Krijger, R. R. (2006). "Childhood Papillary Thyroid Carcinoma with Miliary Pulmonary Metastases".Journal of

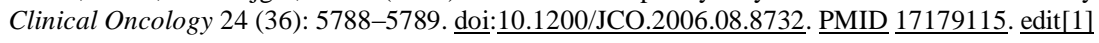

